\title{
Cancer vaccines: pessimism in check
}

\section{To the editor:}

In the September issue of this journal, Rosenberg and colleagues published a turning-point article on the state of the art and future of cancer immunotherapy ${ }^{1}$. The authors report a low tumor regression rate $(2.6-3.3 \%)$ in patients $(n=1,205)$ with metastatic cancer who underwent different types of active specific immunotherapy (ASI), and suggest adoptive transfer combined with nonmyeloablative chemotherapy as the future of cancer immunotherapy.

In the era of molecularly targeted anticancer therapies, we believe that investigation of ASI needs to be supported, although careful study design and use of standardized clinical and immunological criteria are warranted. By contrast, Rosenberg's Perspective risks being perceived by the scientific community as a strong negative message regarding cancer vaccines, which might weaken the enthusiasm surrounding antitumor ASI. In our opinion this would not be justified, as the failure of cancer vaccines for the treatment of metastatic disease - which is so far undeniable-should not discourage the continued investigation of the therapeutic potential of such approaches. Moreover, Rosenberg et al. do not address the issue of why ASI has not met expectations despite the large body of preclinical evidence supporting its rationale.

In an extensive review of the literature $(n=$ $2,933)^{2}$, we found that the overall response rate (tumor disappearance or shrinkage $\geq 50 \%$ ) is $10 \%$, and is likely to be even higher using RECIST criteria adopted by Rosenberg et al. As such, the figures reported by Rosenberg and colleagues seem too pessimistic. It should also be remembered that most patients are heavily treated with conventional chemotherapeutic regimens before undergoing ASI, suggesting that they are likely to be affected with particularly aggressive or resistant tumors as compared to those who undergo first-line chemotherapy.

As in the case of some standard chemotherapeutic regimens ${ }^{3}$, the potential of ASI might be better exploited in the adjuvant rather than in the therapeutic setting. Recent results $^{4,5}$ suggest that ASI might be more effective in the presence of minimal residual disease, owing to a favorable effector/target ratio as compared to the scenario of metastatic disease. Other large trials have shown that ASI effectiveness correlates with a particular immunological setting (e.g., HLA haplotype) of patients or immune response to the vaccination ${ }^{6,7}$, strengthening the idea that the combination of certain tumor or host immune-related features can benefit from therapeutic vaccination. Opposite findings should not be regarded as a proof confuting the above statement, but rather suggest that some immunological parameters commonly utilized for monitoring vaccinated patients are not suitable for the detection of an effective immune response. In this regard, the identification of novel surrogate markers of T-cell activation (e.g., LAMP-1) should be intensely pursued ${ }^{8}$, as should the study of the kinetics of vaccine-driven immune responses. Unlike chemotherapeutic drugs, immunogen efficacy does not follow linear dose-effect kinetics, but rather depends on the complex interplay of many variables (e.g., administration route, minimum effective dose, vaccination schedule, type of immunological adjuvant and host immunological competence).

Rosenberg and colleagues have recently reported promising results in a small set of patients treated with expanded tumor-reactive $\mathrm{T}$ cells ${ }^{9}$. Similar approaches might also be exploited for ASI. In a previous experience of the same research group, the addition of interleukin-2 to peptide-based anti-melanoma vaccination resulted in a high tumor response rate $(42 \%)$. But no correlation between clinical outcome and immune response was observed, and the results have not been replicated in other trials. Nevertheless, recent preclinical advances might pave the way to break tumor immune escape and resistance and allow for an ASI-driven T-cell expansion of therapeutic impact, as illustrated by the following examples.

Novel classes of tumor-associated antigens (TAA) such as xenogeneic homologs of the human TAA, survivin and telomerase, are being successfully used in animal tumor models to overcome the drawback of most TAAs so far utilized for ASI that are weakly immunogenic and do not have a vital role in the metabolism of malignant cells ${ }^{10,11}$.

The blockade of receptors (e.g., CTLA-4), cytokines (e.g., TGF- $\beta$, IL-10) or enzymes (e.g., indoleamine 2,3 dioxygenase) involved in the activity of key cells mediating peripheral tolerance (e.g., T-regulatory cells, tolerogenic dendritic cells) enhances the ability of anticancer vaccination to control tumor growth in preclinical models ${ }^{12-14}$. Yet, recent evidence $^{15}$ suggests that one missing link between the generation of TAA-specific T cells and immune-mediated tumor rejection might be an 'adequate' level of inflammation provided by innate immunity (e.g., natural killer cells). Only the clinical implementation of these and other insights will determine whether the findings reported in animal models can be translated into survival advantage for humans.

The above considerations support our belief that the potential of cancer vaccines is far from being fully exploited and that the preclinical and clinical results achieved so far should be regarded as a stimulus to further dissect the enigma of tumor responsiveness to ASI rather than a substantial failure. The challenging and fascinating task of tumor immunology remains to unravel the molecular and cellular mechanisms underlying a successful immune response, with the aim of reproducing the cascade of biological phenomena conducive to cancer regression in a larger proportion of patients. Although much work remains, to give up on cancer vaccines seems premature.

Simone Mocellin ${ }^{1}$, Susanna Mandruzzato ${ }^{2}$, Vincenzo Bronte ${ }^{2}$, \& Francesco M Marincola ${ }^{3}$

${ }^{1}$ Department of Oncology and Surgical Sciences, Surgery Section, University of Padova, Via Giustiniani, ${ }^{2}, 35128$ Padova, Italy. 2Department of Oncology and Surgical Sciences, Oncology Section, University of Padova, Via Gettamelata, 64, 35128 Padova, Italy. ${ }^{3}$ Immunogenetics Section, Department of Transfusion Medicine, Clinical Center, National Institutes of Health, Bethesda, Maryland 20892, USA.

e-mail:mocellins@hotmail.com 
1. Rosenberg, S.A., Yang, J.C. \& Restifo, N.P. Nat. Med. 10, 909-915 (2004)

2. Mocellin, S., Mandruzzato, S., Bronte, V., Lise, M. \& Nitti, D. Lancet Oncol. 5, 681-689 (2004).

3. Advanced Colorectal Cancer Meta-Analysis Project. J. Clin. Oncol. 10, 896-903 (1992).

4. Jocham, D. et al. Lancet 363, 594-599 (2004).

5. Bystryn, J.C. et al. Clin. Cancer Res. 7, 1882-1887 (2001).

6. Sosman, J.A. et al. J. Clin. Oncol. 20, 2067-2075 (2002).

7. Harris, J.E. et al. J. Clin. Oncol. 18, 148-157 (2000).

8. Rubio, V. et al. Nat. Med. 9, 1377-1382 (2003).

9. Dudley, M.E. et al. Science 298, 850-854 (2002)

10. Nair, S.K. et al. Nat. Med. 6, 1011-1017 (2000).

11. Wei, Y.Q. et al. Proc. Natl. Acad. Sci. USA 98, 11545-11550 (2001).

12. Hodi, F.S. et al. Proc. Natl. Acad. Sci. USA 100, 4712-4717 (2003).

13. Wang, T. et al. Nat. Med. 10, 48-54 (2004).

14. Munn, D.H. \& Mellor, A.L. Trends Mol. Med. 10, 15-18 (2004).

15. Daniels, G.A. et al. Nat. Biotechnol. 22, 1125-1132 (2004).

\section{To the editor:}

In a recent Perspective, Rosenberg and colleagues commented on the need to revise current strategies for therapeutic vaccination against cancer, given disappointing clinical response rates in recent trials ${ }^{1}$. Among 765 patients treated in 35 trials selected to be representative and encompassing of multiple cancer types (see Table 5 in ref. 1), the cumulative objective response rate was only $3.8 \%$. In trials using poxviruses, peptides and modified tumor cells, clinical responses were seen in $0 \%, 4.0 \%$ and $4.2 \%$ of subjects, respectively, and tumor regressions were seen in $7.1 \%$ of patients receiving dendritic cell vaccines.

This analysis does not include a study of dendritic cell-based vaccination for nonHodgkin B cell lymphoma targeting tumor-

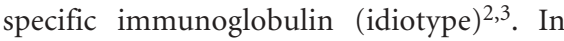
this study, the objective tumor regression rate was $31.6 \%$ among 19 patients with disease evaluable for response by standard radiographic criteria (50\% decrease in the sum of the products of perpendicular diameters of all lesions; no increase in any lesion), including four durable complete tumor regressions. Although this study had a small sample size like others included in the analysis, the $31.6 \%$ objective response rate exceeds all others cited. Given these results, further studies of dendritic cell-based vaccination for lymphoma are now underway, along with several phase 3 randomized studies of idiotype protein vaccines ${ }^{4,5}$.

Non-Hodgkin lymphomas are largely incurable, vascularized cancers that account for over 23,000 deaths annually in the US ${ }^{6}$. Rosenberg et al. suggest that easier access to the circulation and the expression of costimulatory molecules by tumor cells may be a key to the clinical responsiveness of lymphoid tumors. However, other factors also deserve consideration, including the nature of the target antigen. One reason the target is important relates to the mechanism of the antitumor effect. Antibodies are believed to be an important component of the immune response to cell-surface targets such as lymphoma idiotype ${ }^{7}$. The antibody response is prompted through the use of full-length tumor antigen, with $\mathrm{Fc}$ receptor-bearing cells possibly serving as effectors ${ }^{7}$. But durable tumor regressions have also been observed in the absence of measurable anti-idiotype antibodies $^{2-4}$, thereby implicating $\mathrm{T}$ cells as critical effectors in some cases. Thus, although the early clinical experience with adoptive $\mathrm{T}$ cell therapy for melanoma seems promising in contrast to therapeutic vaccine results reported in melanoma to date ${ }^{8}$, this approach may not be required to achieve success against all tumor types.

The diverse biology of human cancers is likely to preclude any single tumor type or approach from serving as a dominant paradigm for the development of immunotherapies successful across a broad spectrum of cancers. Rather, the careful tailoring of particular vaccine, adoptive transfer and antibody strategies to particular antigens (and to particular cancers) may translate into the most fruitful outcomes for cancer immunotherapy.

\section{John M Timmerman ${ }^{1}$ \& Ronald Levy ${ }^{2}$}

${ }^{1}$ Division of Hematology \& Oncology, CHS Room 42-121 University of California, Los Angeles, 10833 Le Conte Avenue, Los Angeles, California 90095-1678, USA. ${ }^{2}$ Division of Medical Oncology, CCSR Room 1105 Stanford University Medical Center, 269 Campus Drive, Stanford, California 94305-5151, USA.

E-mail: jtimmerman@mednet.ucla.edu

1. Rosenberg, S.A., Yang, J.C. \& Restifo, N.P. Nat. Med. 10, 909-15 (2004).

2. Timmerman, J.M. et al. Blood 99, 1517-1526 (2002).

3. Hsu, F.J. et al. Nat. Med. 2, 52-58 (1996).

4. Bendandi, M. et al. Nat. Med. 5, 1171-1117 (1999).

5. Timmerman, J.M. Int. J. Hematol. 77, 444-455 (2003).

6. Jemal, A. et al. CA Cancer J. Clin. 53, 5-26 (2003).

7. Weng, W.K., Czerwinski, D., Timmerman, J., Hsu, F.J. \& Levy, R. J. Clin. Oncol. 23, published online 13 October, 2004.

8. Rosenberg, S.A. \& Dudley, M.E. Proc. Natl. Acad. Sci. USA 101 Suppl. 2, 14639-14645 (2004).

\section{Rosenberg, Yang and Restifo reply:}

Our recent Perspective was based on our nine-year experience with the treatment of 440 patients and a review of the current literature of trials using a variety of cancer vaccine approaches including peptides, recombinant viruses, whole tumor cells, dendritic cells and heat shock proteins ${ }^{1}$. Three points were emphasized in our Perspective:

1. Currently available vaccine approaches have not been effective in mediating cancer regression.

2. In the evaluation of cancer immunotherapy it is essential that objective measures of cancer regression (World Health Organization or RECIST criteria) be used ${ }^{2,3}$.

3. Immunotherapy of cancer based on the adoptive transfer of immune lymphocytes can mediate the reproducible regression of cancer in animal models and in humans ${ }^{4-7}$. We have now tripled the size of our originally reported cohort of patients and we currently observe objective clinical responses in more than $50 \%$ of a group of heavily pretreated patients with metastatic melanoma, many of whom have bulky disease (Dudley et al., $J$. Clin. Oncol., in the press). Based on these ongoing studies, our enthusiasm for the field of cancer immunotherapy has never been greater.

The analysis of cancer vaccines in our Perspective represents a 'reality check' of the current cancer vaccines used alone and not a pessimistic outlook for the future. Although Mocellin et al. agree that the lack of effectiveness of current cancer vaccines is "undeniable," they misinterpret the thrust of our paper when they talk about giving up on cancer vaccine approaches. In fact, as we pointed out, the elegant work of Timmerman et al. showed that dendritic cell vaccines may be effective for the treatment of patients with Bcell lymphomas ${ }^{8}$. Unfortunately, the many reasons for the selective susceptibility of hematopoietic tumors to chemotherapy and immunotherapy approaches, as detailed in the letter by Timmerman and Levy do not seem to apply to tumors of nonhematopoietic cell origin, the subject of our Perspective.

It is especially difficult in clinical studies that involve so much emotional energy and hard work to conclude that one's decade-long efforts have not been effective in helping cancer patients. But it impedes progress and does a disservice to patients if approaches that have been clearly shown to be ineffective continue to be applied.

The letter by Mocellin et al. states that they found a $10 \%$ overall response rate to cancer vaccines. However, many of the studies they included (in their ref. 2) used nonaccepted criteria for clinical repsonse, exactly the kinds of errors that we pointed out in our Perspective that can mislead workers in this 


\section{CORRESPONDENCE}

field. Mocellin et al. also state a common opinion that cancer vaccines would be more effective in the adjuvant setting. This is not supported by the literature. There are few, if any, examples in oncology in which adjuvant interventions are effective when there is no impact in patients with metastatic disease. Clinical studies in the adjuvant setting require large numbers of individuals to be followed for long periods of time. Unfortunately, it simply takes longer to evaluate a particular intervention in the adjuvant setting than by testing it in patients with metastatic disease in whom cancer regression can be rapidly determined.

We remain optimistic that active immunization approaches can be developed that mediate the regression of metastatic cancer. As we described in our Perspective, our own preclinical and clinical efforts to develop active immunization approaches remain strong-we urge that new approaches based on evolving immunologic information be explored. In these studies, however, if claims of patient benefit are made, it is vital that investigators use accepted objective criteria when reporting the results of their clinical trials and acknowledge a lack of efficacy when these criteria are not met.

Our point is not that cancer vaccines cannot be effective, but rather that current vaccines are not effective. The initial success of cell-transfer immunotherapies in mediating the regression of large established cancers in patients with metastatic melanoma show that the immune system can indeed be harnessed to mediate cancer regression ${ }^{4}$. Facing the reality of current vaccine trial results can facilitate future progress in this exciting area of cancer treatment.
Steven A Rosenberg, James C Yang \& Nicholas P Restifo

Surgery Branch, Center for Cancer Research, National Cancer Institute, National Institutes of Health, CRC Room 3-3940, 10 Center Drive, MSC 1502, Bethesda, Maryland 20892-1201, USA.

e-mail:sar@mail.nih.gov

1. Rosenberg, S.A., Yang, J.C. \& Restifo, N.P. Nat. Med. 10, 909-915 (2004).

2. Miller, A.B., Hoogstraten, B., Staquet, M \& Winkler, A. Cancer 47, 207-214 (1981).

3. Therasse, P. et al. J. Natl. Cancer Inst. 92, 205-216 (2000).

4. Dudley, M.E. et al. Science 298, 850-854 (2002).

5. Overwijk, W.W. et al. J. Exp. Med. 198, 569-580 (2003).

6. Klebanoff, C.A. et al. Proc. Natl. Acad. Sci. USA 101, 1969-1974 (2004).

7. Rosenberg, S.A. \& Dudley, M.E. Proc. Natl. Acad. Sci. USA 101, 14639-14645 (2004).

8. Timmerman, J. M. et al. Blood 99, 1517-1526 (2002). 